\title{
Construcción de comunidades colaborativas desde el diseño y el emprendimiento endógeno ${ }^{1}$
}

David Arturo Ospina Ramírez $z^{2}$ Universidad Católica de Manizales david0206ospina@gmail.com

DOI: https://doi.org/10.21158/01208160.n84.2018.1917

Fecha de recepción: 19 de septiembre de 2017

Fecha de aprobación: 02 de febrero de 2018

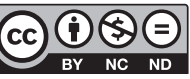

Cómo citar este artículo / To reference this article / Comment citer cet article / Para citar este artigo:

Ospina Ramírez, D. A. (2018). Construcción de comunidades colaborativas desde el diseño y el emprendimiento endógeno. Revista EAN, 84, (pp. 63 -77). DOI https://doi.org/10.21158/01208160.n84.2018.1917

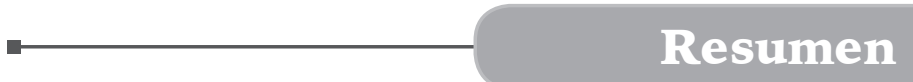

Este artículo visibiliza algunos de los resultados de un proyecto que buscó indagar por el desarrollo de comunidades colaborativas aplicando técnicas del diseño participativo; permitiendo la emergencia de emprendimientos sociales desde la resignificación de lo que implica emprender en comunidades locales, tomando recursos del diseño, el arte y los diálogos generativos. Se centra en los aportes del diseño endógeno, es decir, el diseño que nace desde la misma comunidad y que facilita la construcción de lazos fuertes y colaborativos entre los artesanos, resignificando el emprendimiento, haciendo especial énfasis en los recursos propios de la comunidad, para la identificación de caminos posibles y el fortalecimiento de sus lazos relacionales. La metodología de la investigación se basó en la investigaciónacción participativa, lo que permitió intervenir la comunidad y desde la hermenéutica comprensiva, comprender las maneras en que el diseño y el emprendimiento han favorecido el desarrollo de la misma.

\section{Palabras clave}

Artesanos, diseño endógeno, emprendimiento social, desarrollo endógeno, comunidades generativas.

\footnotetext{
${ }^{1}$ Este artículo resultado de investigación busca evidenciar algunos datos del estudio sobre el diseño endógeno desde el proyecto Artesanos de Marfil de la Fundación Elefantes de Colores, desarrollado en la Manizales (Colombia), entre el 1 de marzo y el 30 de septiembre de 2016, con artesanos locales de la comunidad de la Totuma.

${ }^{2}$ Magister en Educación y Desarrollo Humano, Fundación Cinde y Universidad de Manizales. Magister en Gestión de Proyectos, Universidad EAN y Université du Québec. Docente de Especializaciones en Educación Universidad Católica de Manizales. Líder de proyectos en Fundación Elefantes de Colores. Ganador Fondo Emprender de la Universidad EAN 2015. ORCID: https://orcid.org/0000-0002-1954-2489
} 


\title{
Building up collaborative communities from its design and endogeneous entrepreneurship
}

\begin{abstract}
This article shows some project outcomes resulting from the study of the development of collaborative communities and the application of participatory design techniques. It also allows the emergency of social entrepreneurship by redefining it in local communities and getting resources from design, art and generating dialogues. This study focuses on some contributions from endogenous design, that is to say, the design which resulted from the community itself and which facilitated the establishment of strong ties and collaboration between artisans, redefining entrepreneurship and making emphasis on community owned resources for the identification of possible ways and the fostering of relationship ties. The research methodology used was based on participatory action research which intervened the community and from the perspective of comprehensible hermeneutics to understand different ways in which design and entrepreneurship favored its own development.
\end{abstract}

Key words: artisans, endogeneous design, social entrepreneurship, endogenous development, generating communities.

\section{Construction de communautés collaboratives à partir de la conception et de l'entrepreneuriat endogène}

Résumé. Cet article présente les résultats d'un projet d'étude sur le thème du développement de communautés collaboratives appliquant des techniques de conception participatives permettant l'émergence d'entreprises à caractère social grâce à la reconceptualisation de l'entrepreneuriat dans les communautés locales et utilisant des concepts du design, de l'art et des dialogues génératifs. Cet article analyse plus particulièrement les contributions de la conception endogène, c'est-à-dire de la conception naissant de la communauté elle-même en facilitant la construction de liens collaboratifs forts entre les artisans, en relançant l'entrepreneuriat et en mettant l'accent sur les ressources de la communauté pour identifier les possibilités d'un approfondissement des liens relationnels. La méthodologie de cette étude se base sur le concept de la recherche-action participative ayant permis l'intervention de la communauté et de l'herméneutique globale afin de comprendre comment la conception et l'entrepreneuriat ont favorisé ce développement.

Mots clefs: artisans, conception endogène, entrepreneuriat social, développement endogène, communautés génératives.

\section{Construção de comunidades colaborativas a partir do desenho e do empreendimento endógeno}

Resumo. Este artigo visibiliza alguns dos resultados de um projeto que procurou indagar pelo desenvolvimento de comunidades colaborativas aplicando técnicas de desenho participativo, permitindoaemergência deempreendimentos sociais desde a ressignificação do que implica empreender em comunidades locais, tomando recursos do desenho, da arte e dos diálogos generativos. Este documento se centra nas contribuições do desenho endógeno, isto é, do desenho que nasce desde a mesma comunidade e que facilita a construção de laços fortes e colaborativos entre os artesãos, ressignificando o empreendimento, dando especial ênfase aos recursos próprios da comunidade, para a identificação de caminhos possíveis e o fortalecimento de seus laços relacionais. A metodologia da pesquisa se baseou na pesquisaação-participativa, o que permitiu intervir na comunidade e a partir da hermenêutica compreensiva, entender as maneiras como o desenho e o empreendimento têm favorecido o desenvolvimento da mesma.

Palavras-chave: artesãos, desenhoendógeno, empreendimento social, desenvolvimento endógeno, comunidades generativas. 


\section{Introducción}

Esta investigación visibiliza algunos de - los resultados del proyecto Artesanos de Marfil de la Fundación Elefantes de Colores en Manizales (Colombia). Además, muestra los aportes del diseño participativo y los diálogos generativos como recursos para el fortalecimiento del diseño endógeno, es decir, el diseño y el desarrollo pensado por la misma comunidad que se genera desde sus propios recursos, fortalezas y potencialidades. El proyecto contó con componentes de desarrollo y con componentes de investigación de la investigación-acción participativa, los cuales permitieron intervenir la comunidad desde una postura observadora y generativa e identificar el emprendimiento social como un camino viable para el fortalecimiento de la comunidad. La comunidad de la Totuma se ubica en zonas aledañas a Manizales y está integrada por artesanos de diferentes lugares del país, es dirigida por un taita, que es un adulto mayor que cuenta con saberes ancestrales del sur del país, quien realiza diferentes ceremonias y procesos grupales desde la conexión con la naturaleza. Esta comunidad ha generado diferentes desarrollos colectivos como agrupaciones musicales, un restaurante vegano en Manizales y diferentes proyectos que involucran medicinas ancestrales.

Por otra parte, se enfoca en las capacidades de la misma comunidad para construir nuevos caminos y emprender colaborativamente proyectos que permitan resignificar el emprendimiento y fortalecer los lazos de la comunidad desde el trabajo colaborativo. Así mismo, se ocupa de evidenciar las condiciones que han fortalecido el diseño endógeno en la comunidad y que han permitido resignificar el emprendimiento social desde los criterios locales del colectivo.

Por otro lado, se encontrará un análisis teórico fundamentado, principalmente, en el diseño endógeno y diseño participativo, posteriormente un abordaje sobre el emprendimiento social y uno desde el trabajo colaborativo y los diálogos generativos, una descripción de la ruta metodológica, algunos resultados del proyecto Artesanos de Marfil y las conclusiones que emergen desde la apuesta por el emprendimiento social de la comunidad de la Totuma. 


\section{El emprendimiento social como estrategia de cohesión colectiva}

$\mathrm{E}^{1}$ emprendimiento se ha caracterizado como una ruta clara para el desarrollo de las comunidades y ha sido pensado como una estrategia para garantizar la emergencia de nuevos empleos y nuevas formas de generar recursos para los países, principalmente aquellos que no pueden garantizar empleo a su población, como es el caso de Colombia, país que según GEM Colombia 2014 (Universidad del Norte, 2016), se ubica entre los cinco países más emprendedores del planeta.

El emprendimiento social se caracteriza y se diferencia de los emprendimientos comunes por determinadas características que diversos autores han intentado rescatar desde sus propias prácticas. Global Entrepreneurhip Monitor (2016) explica que normalmente los emprendimientos sociales son desarrollados por jóvenes entre los 18 y los 34 años. Por su parte, Bargsted (2013) señala que el emprendimiento social:

va más allá de generar un negocio rentable en vistas de una oportunidad o necesidad de emprender. Implica elaborar, ejecutar y sustentar iniciativas orientadas a la superación de una dificultad social, y el logro de un beneficio común a un grupo humano, ya sea por medio de actividades empresariales o social-comunitarias. Como otro tipo de emprendimiento, cuenta con los elementos centrales de innovación y riesgo en la solución novedosa a una necesidad o problema. Sin embargo, ya se evidencia contraste entre emprendimiento comercial y social. La principal diferencia propuesta entre ambos tipos de emprendimiento trata sobre la preponderancia de los objetivos sociales y/o ambientales por sobre los económicos (p. 122).
Los emprendimientos sociales, aunque cuentan con los criterios comunes de cualquier ruta de negocio, tienen un interés particular en el medio ambiente, en el desarrollo social $y$, principalmente, en el desarrollo local. Yunus (2011) propone diferentes criterios para validar que una empresa es social, entre ellos, las ganancias de las empresas sociales no se dividen entre sus accionistas, siempre se reinvierten en el negocio o se usan para la creación de nuevos emprendimientos sociales. También postula que los salarios de los colaboradores de este tipo de empresa son mejores que los de empresas de otros tipos, y uno de sus principios más importantes es que las empresas sociales tienen su fundamentación en la búsqueda de soluciones reales a problemas reales de la comunidad y no en la búsqueda de crear necesidades.

En los emprendimientos en países latinoamericanos, gran parte de su economía se fundamente en la artesanía, por ello es importante comprender que el emprendimiento social es mucho más amplio y reúne diversas intencionalidades y condiciones que fortalecen el desarrollo de una comunidad local. Por ejemplo, además de buscar dar solución a necesidades de una comunidad, también se pueden enfocar las intenciones de los emprendedores en dar fortaleza a las oportunidades con las que cuenta la comunidad, transmitir sus conocimientos, cultura, historias y significados que tienen determinados objetos para su comunidad a quienes compran los objetos que se producen. Así mismo, se pueden considerar aspectos como el perfil ambiental de la producción, incluso el ciclo de vida del uso de los productos, los cuales generan a través de sus objetos relaciones sociales más fuertes y alternativas como el 
uso de insumos y materias primas locales que permitan el desarrollo de otras áreas de la misma comunidad.

\subsection{La creatividad como fuente de construcción y fortalecimiento del emprendimiento}

Los emprendimientos sociales, por su naturaleza, implican un acercamiento a la realidad de las comunidades con ciertas posturas de alteridad y altruismo, pero también con fuertes niveles de realismo y comprensión de los fenómenos sociales. Es en esta articulación entre alteridad, altruismo y comprensión de la realidad que el potencial creativo de los emprendedores se despliega. En palabras de Yunus (2011):

La motivación para poner en marcha una empresa social es simple. Comienza con el idealismo y la esperanza que están fuertemente arraigados a los seres humanos. [...] La empresa social ofrece también una salida a la creatividad que millones de personas albergan en su interior (p. 56).

Yunus, expone también lo que se puede entender por creatividad:

Creatividad no tiene que significar algo grande. Puede ser simple, como advertir un problema y pensar «me pregunto si alguien habrá intentado alguna vez resolver el problema haciendo esto» $y$, entonces intentarlo. Una pequeña solución local puede llegar a ser la semilla de una solución global (p. 56).

Es claro que la creatividad es el motor que impulsa nuevas soluciones a problemas determinados. Sin embargo, la creatividad cuenta con unas características importantes para ser consideradas respecto de las acciones que llevan a emprender.
La creatividad es un proceso por el cual dentro de una cultura termina modificado un campo simbólico. La creatividad versa sobre canciones nuevas, ideas nuevas, máquinas nuevas [...]. Para alcanzar la creatividad en un campo ya existente, se ha de disponer de atención sobrante. También parece que los centros de creatividad tienden a estar en la intersección de culturas diferentes, donde creencias, estilos de vida y conocimientos se mezclan y permiten ver a los individuos nuevas combinaciones de ideas con mayor facilidad (Csikszentmihalyi, 1998, p. 23).

Csikszentmihalyi también expone cinco factores clave para definir que algo es creativo: el sujeto que desea emprender un acto creativo debe tener conocimiento del campo, pues no es posible modificar un campo que no se conoce o sobre el cual no se tiene experiencia; también postula que es importante que la solución dada sea mejor a las soluciones preexistentes; y que esta debe ser evaluada y aceptada por expertos en el mismo campo.

En los emprendimientos sociales de carácter local que se basan en la artesanía, la creatividad puede ser entendida desde la producción misma de objetos novedosos o de sistemas producto-servicio que transformen el campo del emprendimiento en este contexto. A diferencia de lo planteado por Csikszentmihalyi, el proyecto Artesanos de Marfil (Fundación Elefantes de Colores, s. f.), comprende la creatividad como un recurso colectivo que implica la transformación de unos recursos y unas condiciones dadas en nuevas formas de construir la realidad de las comunidades.

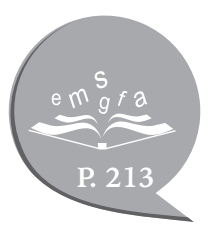




\section{Diseño endógeno como recurso para el emprendimiento y la construcción social}

.

$E^{1}$ diseño endógeno implica abordar dos conceptos: el diseño y el desarrollo endógeno. El desarrollo endógeno:

puede definirse como «desarrollo desde adentro" o desarrollo basado principalmente -aunque no de forma exclusiva- en estrategias, conocimiento, instituciones y recursos locales. Es un proceso continuo de "curación", adaptación e innovación, que comienza desde el interior de la comunidad local. Un criterio clave para el desarrollo endógeno es que es controlado por actores locales. Se dirige hacia el reforzamiento de los recursos locales para el beneficio de las poblaciones locales, y reforzando la habilidad para integrar elementos externos a las prácticas locales. (Rist, 2005, p. 26)

Examinar el desarrollo desde el interior de la comunidad implica pensar que es la comunidad la que elige las rutas de este desarrollo, teniendo en cuenta sus fortalezas, sus capacidades y sus potencialidades. El desarrollo endógeno, desde la mirada de las relaciones sociales y el aprendizaje, parafraseando a Rist (2005), implica reconocer que el conocimiento se construye conjuntamente y todos los actores participan en esta construcción y en el aprovechamiento de este.

Para el emprendimiento, el desarrollo endógeno entonces implica la construcción de un proyecto determinado, donde todos los actores que se ven implicados aportan sus ideas, criterios, capacidades y esfuerzos para su ejecución. Desde el diseño, se podría considerar que las herramientas del arte y de este son similares; sin embargo, Elrhoff (1987) realiza una diferenciación entre estos dos conceptos, manifestando: «Al contrario que el arte, el diseño necesita de un fin práctico y lo encuentra ante todo en cuatro requisitos: ser funcional, significativo, concreto y tener un componente social».

Desde lo anterior, se puede considerar que aplicar al desarrollo endógeno estrategias y aproximaciones desde el diseño implica, por ende, pensar en los aportes prácticos y funcionales de la comunidad, que nacen desde sus criterios, pero que, además, pueden tener una aplicación práctica que permita el fortalecimiento de los mismos procesos creativos.

\subsection{Los aportes del diseño participativo a los cambios sociales y culturales de comunidades de artesanos}

El diseño entonces comprendido como «el proceso de adaptación del entorno objetual a las necesidades físicas y psíquicas de los hombres, de la sociedad» (Löbach, 1976), facilita la comprensión de las capacidades humanas para diseñar, es decir, para transformar las condiciones de un contexto dado a fin de mejorar la interacción del hombre con determinado entorno y contexto.

Estas transformaciones, pensadas desde la comunidad, se adaptan y permean fácilmente las realidades del contexto, interactuando con los agentes de transformación y con el contexto desde la estructuración de nuevas maneras de interpretar el mundo. En las comunidades de artesanos, que se vinculan a conocimientos ancestrales de determinadas culturas, se vinculan estos conocimientos con 
las realidades actuales de las comunidades, lo cual implica la combinación de los conocimientos, las creencias y los intereses del artesano local, con la realidad de contextos cercanos donde se despliegan sus creaciones. Por ello, el trabajo colectivo entre artesanos de determinada comunidad permite que se permeen otros campos y otros contextos desde el intercambio cultural.

Las estrategias del diseño participativo se apropian desde las ciencias sociales, generan nuevas estrategias para acercar al interlocutor externo a la comunidad dada y emplean algunas herramientas del diseño, el arte y otras de las ciencias sociales como las cartografías, los grupos focales, las entrevistas, así como desde el diseño de los productos y servicios de las comunidades. Respecto del desarrollo endógeno, se considera que «cada dominio tiene diversos proyectos y subproyectos, y toma en cuenta conceptos generales, tales como sostenibilidad, género y empoderamiento de los actores locales» (Delgado, Tapia y Lisperguer, 2005, p. 34).

\subsection{El diseño participativo y el dialogismo generativo}

El diseño participativo implica la transformación de unos recursos y una realidad dada en una respuesta diferente de lo preexistente y permite que nuevas rutas se tracen desde el interior de las mismas comunidades, donde los externos aportan y fortalecen posibilidades, pero donde priman las elecciones de la comunidad en las rutas del diseño de su desarrollo. Para generar el diseño participativo, se apropian recursos de las ciencias sociales, como se ha mencionado, los cuales emplean fuertemente el dialogismo generativo como recurso mediador. Fried Schnitman (2012) concibe:
La perspectiva creativa del tiempo, el caos como fuerza potencialmente innovadora, la complejidad como un mundo abierto de posibilidades, la construcción activa de sujetos en contexto, de significados y prácticas, el particular recorte de sus realidades y la consideración del conocimiento como un proceso generativo-constructivo, son recursos de los nuevos paradigmas que permiten un desplazamiento desde perspectivas asociadas con un mundo ordenado y predecible a perspectivas en las que la turbulencia, la oscilación, los eventos inusuales o únicos y la innovación conforman oportunidades y posibilidades tanto para la ciencia y las prácticas profesionales como para la vida cotidiana (p. 3).

La mirada generativa implica reconocer unas problemáticas dadas en una comunidad para posicionarse desde las potencias de su contexto, su comunidad y sus individuos y, principalmente, desde las potencialidades de sus relaciones colectivas. El dialogismo generativo implica por medio del diálogo encontrar aquellos recursos y capacidades individuales y colectivos, para la creación de nuevas posibilidades de creación dialógica. En palabras de Fried Schnitman (2012):

Podemos denominar creación dialógica a la construcción gradual en el tiempo de algo nuevo mediante el diálogo reflexivo y el aprendizaje conversacional en grupos humanos. En el desarrollo del proceso las personas o grupos llegan a ver, experimentar, describir, vincularse y posicionarse de una manera diferente. Este enfoque centrado en los diálogos generativos considera a la creación de significado, la experiencia y el conocimiento como procesos constructivos en los que los acontecimientos específicos, los actos y episodios tienen la capacidad potencial de transformar las pautas de relación social desde su interior (p. 4). 
Por tanto, la creación dialógica no es otra cosa que la resignificación de determinados aspectos de la comunidad, por medio del diálogo apreciativo, y por qué no, del diseño apreciativo, es decir, la transformación de unos elementos dados, en unos nuevos, considerando las potencialidades de la comunidad para dar respuestas a las problemáticas dadas.

\section{La creatividad colectiva como escenario de desarrollo local}

C aro (2017) propone que el desarrollo urbano se ve impulsado por los emprendimientos de carácter sostenible e innovador. Por su parte, Ospina-Ramírez y Ospina-Alvarado (2017) proponen que los seres humanos cuentan con un recurso propio y colectivo que es el potencial creativo para la transformación, es decir, la capacidad de tomar unos insumos dados y transformarlos en un resultado diferente de lo existente. Chirinos y Pérez (2016) proponen que en los contextos educativos la gran responsabilidad de las instituciones es la promoción del empoderamiento social desde el emprendimiento de carácter

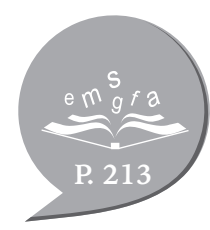
social y sostenible, argumento que soporta Ocampo (2016) al afirmar que la formación integral de los sujetos implica una mirada social de las problemáticas que se presentan en determinado contexto.

El acto de la creación se puede agenciar de manera individual o colectiva, sin embargo, el impacto de las acciones sociales es lo que favorece la potenciación de los recursos de determinada comunidad y así promover el desarrollo de determinados territorios y de las comunidades que los habitan. Parafraseando a Marulanda y Morales (2016), los emprendimientos deben surgir de las necesidades y oportunidades del contexto. Este análisis contextual implica una mirada crítica del emprendedor, quien necesariamente debe construir relaciones simbióticas con la comunidad, y es allí donde se gestan proyectos articulados y con mayor estructura social que les permitan tener sostenibilidad.

\section{Metodología de trabajo}

$\mathrm{E}^{1}$ proyecto fue desarrollado desde la perspectiva de la investigación-acción participativa, lo cual permitió contar con dos macrocomponentes. Desde la gestión del proyecto como desarrollo social, se llevó a cabo bajo los estándares del PMIA Guide to the Project Management Body of Knowledge: $\mathrm{PMBOK}^{\circledR}$ Guide del Project Management Institute (PMI, 2013), lo cual implicó atravesar cinco fases: iniciación, planificación, monitoreo, y control y cierre, además de abordar 13 áreas del conocimiento de la gestión de proyectos: calidad, stakeholders, recursos humanos, tiempo, cambios, costos, comunicaciones, adquisiciones, alcance e integración de todos los procesos y las fases previamente mencionados. Estos permitieron lograr la culminación del proyecto con el cumplimiento a satisfacción de cada uno de sus entregables. 
Desde el punto de vista investigativo, el proyecto asumió la investigación-acción participativa en un estudio de caso de una comunidad particular, desde un enfoque cualitativo, hermenéutico, comprensivo y crítico transformador. Lo anterior implica la participación de la comunidad como un miembro activo dentro de la investigación, haciendo énfasis en las potencialidades y los recursos de los miembros y permitiendo su participación como investigadores activos de su mismo proceso. Es un estudio de caso teniendo en cuenta que el proyecto siguió una ruta de gestión y de investigación con una comunidad específica en Manizales; por tanto, si bien podría ser replicable la estrategia, no existe seguridad de que se obtengan los mismos resultados e impactos. Por su parte, la hermenéutica comprensiva, crítica y transformadora permite dar importancia a las voces de las experiencias de los participantes y lograr un ejercicio de investigación que permita develar una realidad específica, y a su vez, participar activamente en la transformación de la comunidad (Arias y Alvarado, 2015).

\section{Principales experiencias del proyecto Artesanos de Marfil}

$\mathrm{P}$ ara conocer el detalle de las experiencias generadas en el proyecto Artesanos de Marfil, se detalla a continuación su alcance. El proyecto busca fomentar las habilidades artísticas, creativas y proyectivas de jóvenes artesanos de Manizales, con el fin de transformar sus comunidades y construir escenarios conjuntos donde el arte, el diseño y la creatividad puedan posibilitar nuevos caminos desde proyectos sostenibles que puedan ser ejecutados por ellos mismos (Fundación Elefantes de Colores, s. f.).

El grupo de participantes que se integró al proyecto fueron jóvenes entre los $20 \mathrm{y}$ 34 años, que antes de hacer parte de este, contaban con emprendimientos independientes en los cuales desarrollaban diferentes tipos de productos como medicinas con plantas, accesorios en madera o tejidos, decoración para el hogar basada en textiles, productos de higiene personal y de baño a base de plantas y otros recursos naturales.
El proyecto constó de las siguientes fases: primero, se desarrolló un proceso de vinculación y reconocimiento de la comunidad y de los emprendimientos individuales que cada uno de ellos desarrollaba; segundo, se llevó a cabo un mapeo de las expectativas de la comunidad al emprender y una estructuración básica de cada ruta de negocio; tercero, se identificaron los aspectos clave de cada emprendimiento que cumplían con algunos de los criterios de los emprendimientos sociales; cuarto, se definió una ruta de trabajo colectivo en la que todos los artesanos participaron desde sus capacidades y potencialidades; quinto, se hizo un acompañamiento en las mejoras de sus productos desde un perfil de sostenibilidad, donde se consideraron los factores de impacto social, ambiental y económico de sus productos; y sexto, el proyecto concluyó con el montaje de una galería colectiva ubicada en Manizales, en la que todos los productos del colectivo son expuestos de manera alternativa, en busca de compartir con los visitantes las maneras en que los artesanos interpretan el emprendimiento y comparten sus conocimientos ancestrales y su cultura. 


\subsection{El significado de emprender}

El proyecto permitió el mejoramiento de sus productos en términos estéticos y funcionales, así como de sostenibilidad y funcionalidad. El emprendimiento para la comunidad tiene un significado diferente, que si bien no cumple con todos los criterios de sostenibilidad propuestos por Yunus (2012), sí aportan una resignificación de este término en el contexto local (Figura 1).

Figura 1. Taller de creación

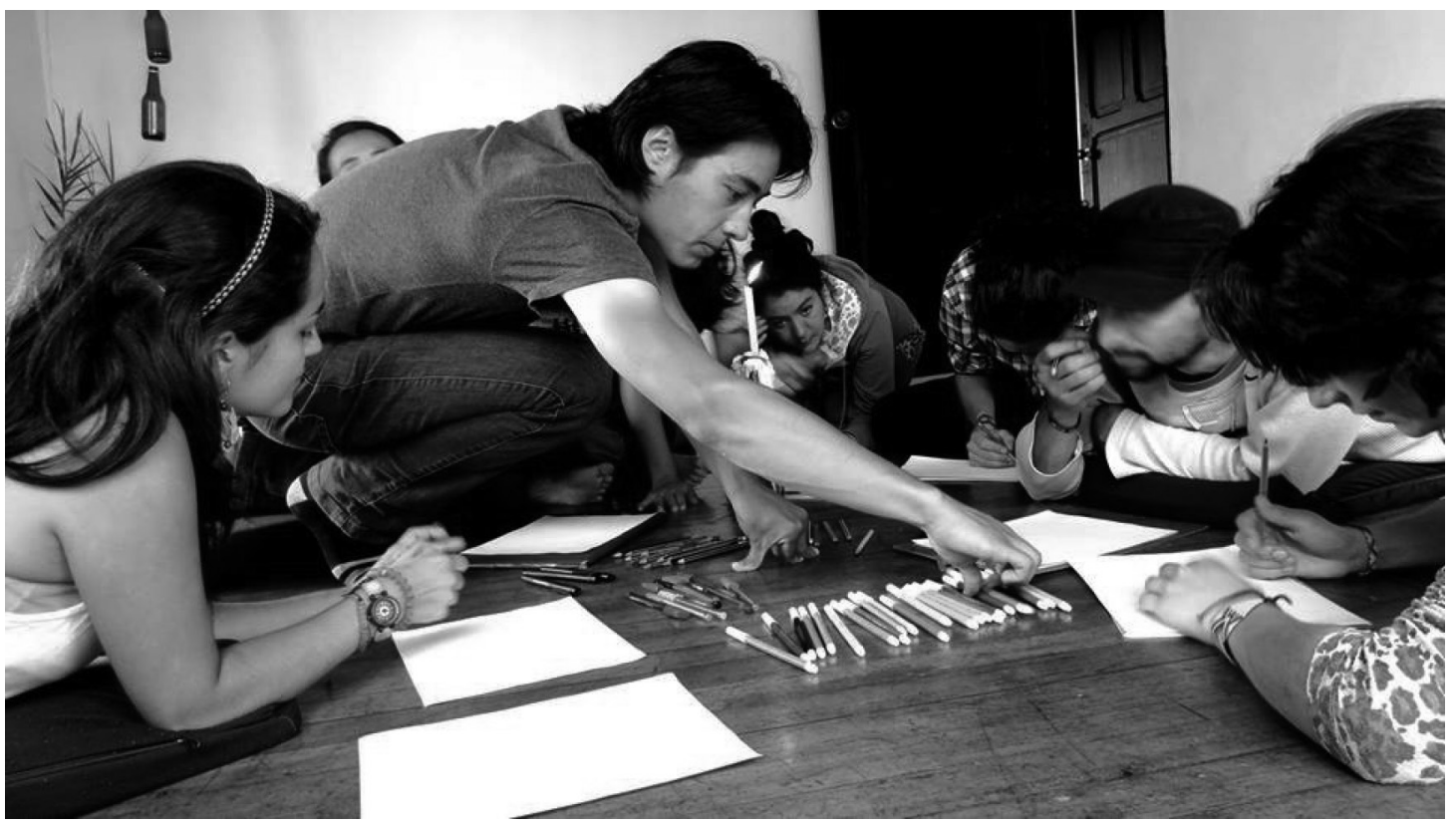

Fuente. Fundación Elefantes de Colores (s. f.).

Emprender para mí implica entregar una parte de mí, de mi historia, de mis conocimientos, intento que mis productos sean naturales y que tengan algo de nuestra cultura; también intento que mis productos toquen de alguna forma a las personas, que les ayuden a ver el mundo de manera diferente, despertando su sensibilidad (Colina, comunicación personal, marzo de 2016).

En este relato de un artesano que produce arte, piezas decorativas y accesorios con materias primas recicladas, se puede identificar que el emprendimiento para él implica imprimir en cada uno de sus desarrollos su cultura y sus conocimientos; el objetivo de cada producto es despertar la sensibilidad de quien adquiere el producto, lo cual se traduce en permitir que el usuario tenga la capacidad de very percibir el mundo de manera diferente. Una de las artesanas expresa:

Emprender para mí es un acto de libertad, me permite desligarme de las dinámicas del mundo económico como lo conocemos hoy, me permite transmitir sanación por medio de productos que nos brinda la naturaleza, encontrando nuevas maneras de contactar y enlazar con la gente (Santacoloma, comunicación personal, marzo de 2016).

El emprendimiento hace posible a los artesanos vivir su cultura sin permitir que se desdibujen sus creencias o maneras de interpretar el mundo. También es claro para 
el colectivo generar proyectos que posibiliten llevar a la sanación o a la transformación, al considerar que sus productos tienen propiedades particulares que les permiten producir transformaciones de diversos tipos en quienes les dan uso.

Emprender me ha permitido unirme a otros emprendedores, me ha permitido generar lazos, también ha sido un proceso sanador, porque, mientras hago cada cosa que mi emprendimiento requiere, pienso que me estoy construyendo, que hace parte de mí y que de alguna forma hace parte del universo; por eso, lo hago con amor y entrega (Luna, comunicación personal, marzo de 2016).

La cosmovisión de los emprendedores evidencia las diferencias que tienen los emprendimientos locales de las rutas de emprendimiento tradicional; de hecho, este se convierte en una manera de desplegar sus propias maneras de concebir el mundo, de vivirlo y de compartirlo con otros.

\subsection{El fortalecimiento de la comunidad}

Un poco más adelante (Figura 2), se evidencia uno de los ejercicios planteados desde el ejercicio investigativo, que consistió en la creación de un futuro soñado de manera colectiva donde los emprendimientos individuales alimentaban la posibilidad de una comunidad unida por medio de un sistema productivo y un ecosistema de emprendimiento sostenible y raizal de la comunidad de la Totuma. Los lazos relacionales de la comunidad se vieron impactados por la construcción de un emprendimiento colectivo que reúne diferentes emprendimientos individuales.

Figura 2. Taller de creación

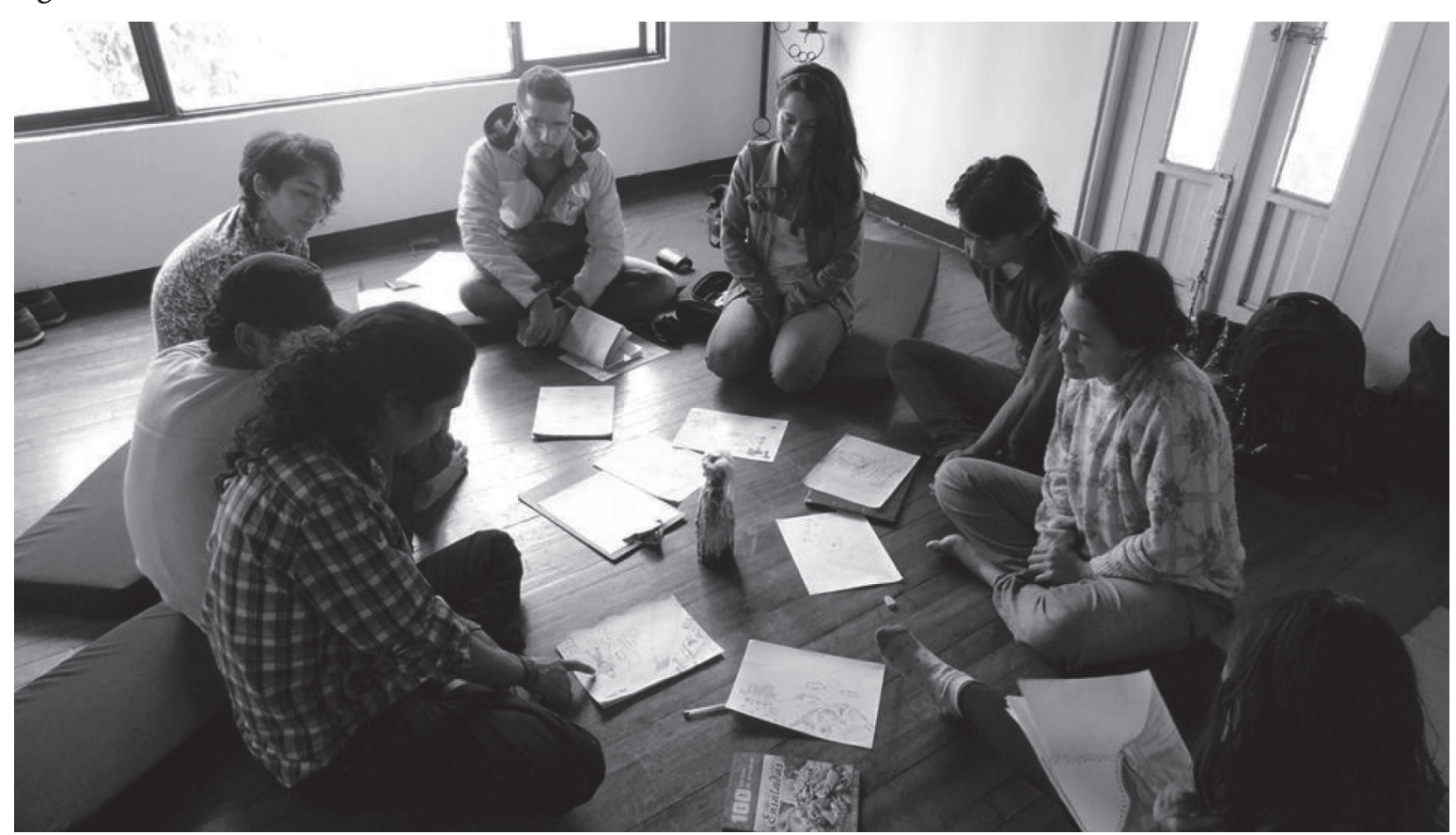

Fuente. Fundación Elefantes de Colores (s. f.). 
Emprender juntos ha facilitado el proceso del emprendimiento, también nos ha permitido entre todos encontrar las necesidades reales que vamos a resolver como comunidad, trabajando conjuntamente desde nuestras fortalezas, desde nuestra unión (Santacoloma, comunicación personal, marzo de 2016).

Emprender colectiva y colaborativamente, en efecto, facilita los procesos que implican este tipo de proyectos, agilizan el posicionamiento y la difusión del colectivo, permiten encontrar las fortalezas y los saberes de una comunidad, potenciarlos desde el trabajo colectivo y articular esfuerzos y conocimientos sobre determinados abordajes a los problemas cercanos a la comunidad.

Siempre quisimos emprender, siempre quisimos materializar esta idea, pero no encontrábamos las maneras de construirlo, luego nos unimos y fue muy sencillo caminar juntos. Lo que a mí me falta lo tienen ellos, y lo que ellos necesitan tal vez yo lo pueda aportar. Es decir, se ha generado una sinergia como grupo (Galvis, comunicación personal, marzo de 2016).

Las experiencias de emprendimiento, individuales y colectivas permiten que la identificación de debilidades y fortalezas como comunidad se haga evidente de manera crítica pero apreciativa y aporte desde los lazos relacionales para el desarrollo de la comunidad por medio del proyecto conjunto.

\subsection{La experiencia del diseño como mediador de identidades colectivas}

El arte y el diseño como actividades proyectuales se emplearon continuamente, desde las actividades de cada uno de los talleres hasta la construcción colectiva de productos y el diseño general de la galería de arte; un factor importante, como el que se evidencia en la fotografía, fue que los diseños que hicieron los artesanos representaron su cosmovisión del mundo, el cual tiene fuerte relación con prácticas ancestrales del territorio. Los diferentes emprendimientos de los artesanos, que finalmente se unieron en el colectivo Artesanos de Marfil, contaron con diferentes tipos de productos que se vieron influenciados por la experiencia del diseño, desde el de alimentos, hasta el artesanal de piezas de uso personal y de uso decorativo. La construcción de cada producto contó con una etapa de diseño colectivo en la que se definieron características funcionales y estéticas. Sin embargo, las intencionalidades de cada artesano se vieron influenciadas por intereses particulares y por sus maneras diversas de concebir el mundo. Parafraseando a Giménez (2005), las identidades colectivas son aquellas que implican un emocionar similar en los integrantes de determinada comunidad, quienes convierten sus acciones en movimientos de mayor impacto que logran afectar a comunidades próximas (Figura 3). 
Figura 3. Ejercicio de indagación apreciativa del contexto

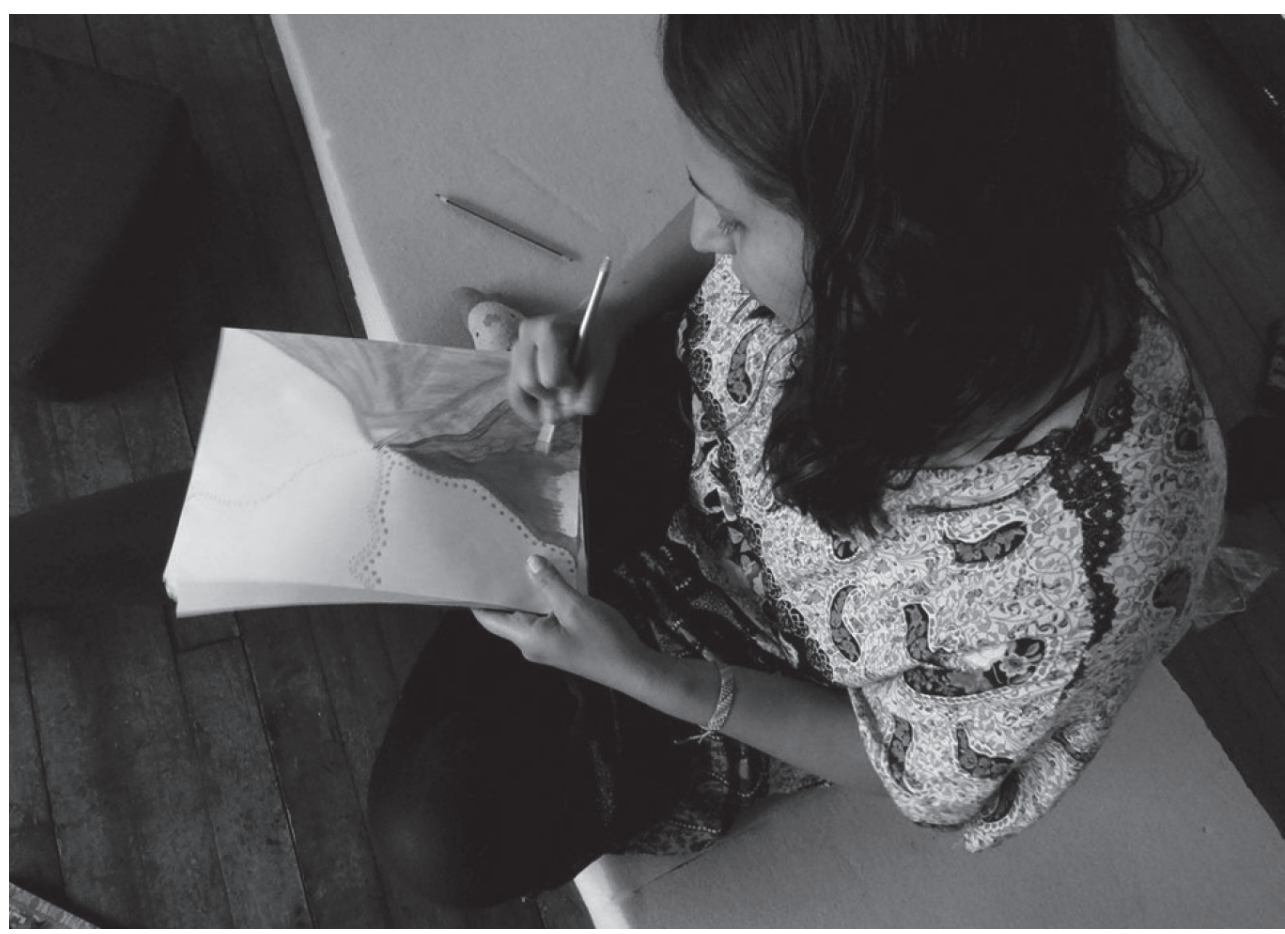

Fuente. Fundación Elefantes de Colores (s. f.).

Las experiencias de diseño generaron en los participantes del proyecto objetivos comunes y emociones identitarias, que permitieron imprimir en la comunidad próxima las maneras de interpretar el mundo y de construir la realidad de la comunidad de la Totuma. Giménez (2005) expresa:

La identidad se define por sus fronteras [...]. a pesar de todo lo este, la identidad de los actores sociales no se define por el conjunto de rasgos culturales que en un momento determinado la delimita y distingue de otros actores. Por ejemplo, un grupo étnico puede adoptar rasgos culturales de otros grupos, como la lengua y la religión, y continuar percibiéndose (y siendo percibido) como distinto de los mismos. Por lo tanto, la conservación de las fronteras entre los grupos étnicos no depende de la permanencia de sus culturas (Giménez, 2005, p. 18).
La identidad del colectivo de la comunidad de la Totuma se vio permeada por una nueva identidad que imprimió el proyecto Artesanos de Marfil. La experiencia de diseño permitió la interacción cultural entre el equipo del proyecto y la comunidad próxima, y facilitó la apropiación por parte y parte de nuevas maneras de interpretar y vivenciar la realidad. En palabras de Alvarado y Ospina (2014), la identidad y la subjetividad de los individuos se constituyen en las relaciones que entraman con los otros; desde allí se configura una identidad colectiva que les permite diferenciar un emprendimiento de otro, pero añadir valor desde el trabajo mancomunado. Los emprendimientos y las experiencias de diseño se vieron influenciados por los factores culturales de la comunidad próxima, y de igual forma, la comunidad se vio influenciada o impactada por los resultados de la experiencia de diseño que se materializaron en productos y en una galería cultural y artesanal. 


\section{Conclusiones y comentarios finales}

$E_{\text {des }}^{1}$ diseño endógeno, desde la perspectiva del diseño permeada por aportes de las ciencias sociales, permite que las comunidades identifiquen estrategias y recursos propios para transformar sus realidades $y$ las realidades de comunidades cercanas. Las estrategias del diseño participativo se integran al dialogismo generativo, pues desde la identificación de potencias y capacidades de las comunidades o colectivos de trabajo colaborativo, fortalecen los lazos relacionales y generan transformaciones de determinados insumos dados para la construcción de resultados diferenciales a las respuestas existentes en sus contextos.

En el trabajo con comunidades artesanales, es importante tener en cuenta que el emprendimiento no es una ruta de negocio o de desarrollo como se ha concebido en el mundo capitalista tradicional; por el contrario, la cosmovisión de las comunidades de este tipo les lleva a buscar el desarrollo del ser humano en otras dimensiones y a resignificar el emprendimiento como un camino para el desarrollo colectivo de la comunidad, donde todos sus miembros imprimen sus potencias y capacidades para la construcción de nuevos caminos diferentes de los conocidos.
El emprendimiento social y el diseño participativo se integran desde las miradas generativas y reconocen las problemáticas de un contexto, pero dando relevancia a las capacidades locales para dar respuestas adecuadas a las realidades de este contexto. Elobjeto, por su parte, en el emprendimiento social, es un mediador social y cultural que permite la generación de identidad entre el productor local y el usuario de su producto, pues, en cada producción, se imprimen características únicas que evidencian las maneras de interpretar el mundo de quien produce cada objeto. El intercambio cultural se ve mediado por las relaciones sociales y el fortalecimiento de sus lazos.

El pensamiento proyectual o el pensamiento de diseño tiene una característica que se evidenció en el trabajo de campo, por ejemplo, desde las relaciones empáticas de la comunidad y desde el cuidado del otro en el trabajo colectivo, y en las acciones transformadoras que trascienden el sentido económico del acto de emprender, que se ubican en unas nociones de desarrollo distintas de las que generan los modelos económicos tradicionales. 


\section{Referencias}

Alvarado, S. y Ospina, H. F. (2014). Configuración de la subjetividad política. Bogotá: Siglo del Hombre.

Arias Cardona, A. M. y Alvarado Salgado, S. V. (2015). Investigación narrativa: apuesta metodológica para la construcción social de conocimientos científicos. Revista CES Psicología, 8(2), 171-181.

Bargsted, A. (2013). El emprendimiento social desde una mirada psicosocial. Civilizar Ciencias Sociales $y$ Humanas, 13(25), 121-132.

Caro Moreno, J. C. (2017). La innovación urbana como factor de desarrollo socioeconómico. Revista EAN, $82,165-178$.

Chirinos Araque, Y. V. y Pérez Peralta, C. M. (2016). La responsabilidad social universitaria: emprendimiento sostenible como impacto de intervención en comunidades vulnerables. Revista EAN, 81, 91-110.

Csikszentmihalyi, M. (1998). Creatividad: el fluir y la psicología del descubrimiento y la invención. Barcelona: Paidós.

Delgado Burgoa, F., Tapia Ponce, N. y Lisperguer, G. (2005). Revitalizando el conocimiento indígena para el desarrollo endógeno. Revista COMPAS, 7, 3437.

Elrhoff, M. (1987). Exposición de arte contemporáneo. Alemania: Kassel, documenta 8. Recuperado de: https://www.documenta.de/es/retrospective/ documenta $8 \#$

Fried Schnitman, D. (2012). Diálogos generativos. Recuperado de https://www.taosinstitute.net/ Websites/taos/files/Content/5695612/DSDialogos_Generativos.pdf

Fundación Elefantes de Colores (s. f.). Proyectos. Recuperado de http://www.elefantesdecolores.org/ fundacion/proyectos/
Giménez, G. (2005). Cultura, identidad y metropolitanismo global. Revista Mexicana de Sociología, 67(3), 483-512.

Global Entrepreneurhip Monitor (2016). Emprendimiento social: reporte especial. Recuperado de http:// gemcolombia.org/publications/emprendimientosocial-reporte-especial/

Löbach, B. (1976). Diseño industrial. Recuperado de http://www.hicistelclick.com/a-que-juega-eldiseno/que-es-el-diseno/

Marulanda Valencia, F. Á. y Morales Gualdrón, S. T. (2016). Entorno y motivaciones para emprender. Revista EAN, 81, 12-28.

Ocampo Eljaiek, D. R. (2016). El emprendimiento social en la formación integral. Revista EAN, 81, 175-190.

Ospina-Ramírez, D. A. y Ospina-Alvarado, M. C. (2017). Futuros posibles, el potencial creativo de niñas y niños para la construcción de paz. Revista Latinoamericana de Ciencias Sociales, Niñez y Juventud, 15(1), 175-192.

Project Management Institute (2013). PMIA Guide to the Project Management Body of Knowledge: $P M B O K^{\circledR}$ Guide. Project Management Institute.

Rist, S. (2005). Desarrollo endógeno como un proceso social. Revista COMPAS, 7, 26-29.

Universidad del Norte (2016). GEM Colombia 2014. Bogotá: Universidad del Norte. Recuperado de http://gemcolombia.org/wp-content/uploads/ GEM-Colombia-2014-versi\%C3\%B3n-digital.pdf

Yunus, M. (2011). Las empresas sociales. Buenos Aires: Paidós. 